\title{
APLICAÇÃO DE SISTEMA MULTICANAL NA DETECÇÃO DE QUEDAS PARA IDOSOS
}

\section{Jocélio Barbosa Ventura}

Mestre em Engenharia Biomédica, pela Universidade de Mogi das Cruzes, São Paulo, Brasil. Engenheiro Elétrico e Eletrônico pela Universidade de Mogi das Cruzes, SP, Brasil. joceliob@gmail.com

\section{Leonardo Juan Ramírez López}

Professor Doutor da Universidade Militar Nueva Granada, Bogotá, Colômbia. Diretor do Grupo de pesquisa em Telemedicina-TIGUM-UMNG, Bogotá Colômbia. Doutor em Engenharia Biomédica pela Universidade de Mogi das Cruzes, São Paulo, Brasil. Mestre em Engenharia de Sistemas pela Universidade Nacional da Colômbia.

leonardo.ramirez@unimilitar.edu.co

\section{Daniel Gustavo Goroso}

Graduação em Bachelerado Universitario (1992) e Licenciatura Plena em Física (1994) pela Universidad Nacional de Tucumán - Argentina. Mestrado em Engenharia Elétrica pela

Universidade de São Paulo (1999). Doutorado em Engenharia Elétrica (orientação Biomédica) pela Universidade de São Paulo (2004). Atualmente é Coordenador de Pesquisa e Desenvolvimento do Instituto de Medicina Física e Reabilitação (IMREA) do HC-FMUSP. Professor/pesquisador da Universidade de Mogi das Cruzes. daniel.goroso@hc.fm.usp.br

\section{RESUMO}

Os recentes avanços tecnológicos oferecem várias opções para a monitorização clínica dentro de uma grande área de cobertura. Esta descoberta ajuda a realizar a supervisão regular de saúde. Um sistema multicanal de telemetria foi desenvolvido através deste estudo de pesquisa a ser aplicada ao monitoramento de três sinais fisiológicos: frequência cardíaca, aceleração e temperatura do corpo. Uma placa eletrônica foi desenvolvida, para captar, pré-processar, armazenar e enviar sinais para um repositório central de dados que nos permite compartilhar dados processados para o monitoramento, medição e controle. $\mathrm{O}$ processo de validação da aplicação foi à detecção da intenção de queda utilizando acelerômetro tri-axial. A aplicação validada, mostrou-se auxiliar para o monitoramento de queda de idosos e para a prevenção e controle de doenças não-transmissíveis (NCD). Os resultados mostraram que as quedas foram detectadas durante a diminuição repentina no nível de tensão 1500-960 mV, enquanto que a sensibilidade do acelerômetro foi ajustada para $1,5 \mathrm{~g}(800 \mathrm{mV})$. Além disso, duas condições obrigatórias devem ser cumpridas para qualquer queda ser detectada: i) atenuação $-530 \mathrm{mV}$ dentro do vetor magnitude de aceleração (aproximadamente 1g), e ii) atenuação anterior deve ocorrer dentro de um período de tempo não superior a 50 milésimos de segundo. Em suma, o sistema de biotelemetria descrito acima é prático, não-invasivo, versátil e acessível, permitindo o uso generalizado no seio da sociedade em geral.

PALAVRAS-CHAVE: multicanal, controle, quedas, idosos. 


\title{
MULTI-CHANNEL APPLICATION SYSTEM OF DETECTION FALLS FOR ELDERLY PEOPLE
}

\begin{abstract}
Recent technological advances provide multiple options for clinical monitoring within a wide coverage area. This breakthrough helps perform regular healthcare oversight. A Multi-Channel Telemetry System was developed through this research study to be applied to the monitoring of three physiological signals: heart rate, movement and body temperature. An electronic board was developed which collects, pre-processes, stores and sends signals to a Central Data Repository which allows us to share processed data for monitoring, measurement and control. The validation process was mean to fall detection application using tri-axial accelerometry. The validated application proved itself ancillary to elderly people fall monitoring and the prevention and control of non-communicable diseases (NCD). The results showed that falls were detected during the sudden decrease in voltage level from 1500 to $960 \mathrm{mV}$, while the sensitivity of the accelerometer was set to $1.5 \mathrm{~g}(800 \mathrm{mV})$. Besides, two mandatory conditions must be met for any falls to be detected: i) $-530 \mathrm{mV}$ attenuation within the acceleration magnitude vector (approx. 1g), and ii) previous attenuation must take place within a period of time not exceeding 50 milliseconds. To sum up, the Biotelemetry System described above is practical, noninvasive, versatile, and affordable allowing for widespread use within the society at large.
\end{abstract}

KEYWORDS: multi-channel, control detection falls, elderly.

\section{APLICAÇÃO DE SISTEMA MULTICANAL NA DETECÇÃO DE QUEDAS PARA IDOSOS}

\section{INTRODUÇÃO}

Neste trabalho a biotelemetria é uma aplicação da telemetria, onde são transmitidos dados biológicos provindos de dispositivos remotos (sensores, placas, módulos, etc.) até uma central de captação de informações para o monitoramento, medição e controle dos mesmos. Atualmente a comunidade cientifica de saúde prefere o termo telemedicina [1], tudo no interesse do avanço da saúde do individuo e de sua comunidade. Entre os anos de 1950 e 2000 a população acima de 75 anos dobrou, esse número saltará em 2030 para aproximadamente 300 milhões de pessoas, dobrando novamente só que em bem menos tempo, o que demonstra uma aceleração na taxa de crescimento deste seguimento da população a partir da década de 80 [2]. Este fato se deve ao aumento da expectativa de vida das pessoas que passaram a ter melhores condições de vida e acesso aos serviços de saúde. De fato, são temas de constante pesquisa: as distâncias alcançadas, quantidade de informação transmitida, área de cobertura e segurança das informações.

Em trabalhos similares, apresenta-se o monitoramento para três sinais fisiológicos: eletrocardiografia, temperatura e saturação de oxigênio; conectados através de uma rede sem fio ZigBee [3,4]; baseados na rede sem fio Bluetooth ${ }^{\mathrm{TM}}$ para conexão à rede GSM celular ou computador [5]. No autocontrole da saúde, ou no auxilio de diagnóstico para avaliar terapias através da análise dos dados coletados.

Atualmente o governo Brasileiro faz inúmeras campanhas para motivar a população no autocuidado da saúde, mediante o uso de uma infraestrutura de comunicação básica, sem 
atrapalhar as atividades diárias de cada pessoa. Neste contexto, este trabalho atinge esta política de governo através de uma ferramenta de biotelemetria que permite o monitoramento, para casos como o controle de quedas. O sistema multicanal desenvolvido foi testado tanto em ambientes controlados (laboratório) para avaliar as informações transmitidas desde a rede de sensores e o dispositivo de comunicação até uma central de captação (computador) que permite armazenar e compartilhar os dados, e em ambiente aberto (fora de laboratório) para avaliar o controle de quedas com idosos e descarregar os dados na central de captação.

\section{MATERIAIS}

Computador desktop: sistema operacional Windows 7, RAM 2 GB, placa de vídeo de 128 $\mathrm{MB}$, velocidade do processador $1,8 \mathrm{GHz}$ e $250 \mathrm{~GB}$ de espaço no disco rígido. Osciloscópio digital: $60 \mathrm{MHz}$, com taxa de amostragem de $2 \mathrm{GS} / \mathrm{s}$ em quatro canais, base de tempo dupla, memórias de formas de onda, porta de comunicação serial (GPIB) e entrada USB, marca Tectronix (Beaverton, USA). Plataforma Arduino BT: plataforma de hardware, com módulo de porta Bluetooth ${ }^{\mathrm{TM}} 2.1$, suporte de entrada/saída incorporado, processador ATmega168, e linguagem de programação aberto padrão (baseado $\mathrm{C}++$ ).

Simulador de Eletrocardiografia: usado para obter o sinal de ECG e evitar conectar os eletrodos ao indivíduo. O simulador tem opção de seleção de 16 formas de onda, indicador de bateria fraca, sistema pré-calibrado do fabricante (QSP Tecnologia, SP-Brasil).

Para selecionar os sensores foi desenvolvida uma matriz de decisão, que consiste em definir os pesos para cada critério de seleção e a qualificação entre 1 e 4 para cada característica técnica do componente, sendo 1: muito ruim, 2: ruim, 3: bom e 4: muito bom. O amplificador de instrumentação foi escolhido para adquirir o sinal de ECG. A seleção foi feita entre três amplificadores disponíveis no mercado: INA115, INA114 e AD620. Na Tabela 1, são apresentados os critérios de seleção, os pesos e os resultados para cada componente.

Tabela 1: Parâmetros escolha sensor AI.

\begin{tabular}{|l|c|c|c|c|c|c|c|}
\hline \multicolumn{1}{|c|}{ SENSOR } & Vr & \multicolumn{2}{c|}{ INA115 } & \multicolumn{2}{c|}{ INA114 } & \multicolumn{2}{c|}{ AD620 } \\
\hline \multicolumn{1}{|c|}{ Critério } & $\mathbf{\%}$ & Valor & Peso & Valor & Peso & Valor & Peso \\
\hline Offset $[\mu \mathrm{A}]$ & 20 & 50 & 0,8 & 50 & 0,8 & 50 & 0,8 \\
\hline CMRR $[\mathrm{dB}]$ & 20 & 115 & 0,8 & 115 & 0,8 & 110 & 0,6 \\
\hline $\mathrm{I}_{\text {Quiescente }[\mathrm{mA}]}$ & 10 & 3 & 0,3 & 3 & 0,3 & 1,3 & 0,4 \\
\hline Encapsulado & 40 & D-DIP & 1,6 & D-DIP & 1,6 & S-DIP & 0,8 \\
\hline Custo & 10 & $20-30$ & 0,3 & $10-20$ & 0,4 & $10-20$ & 0,4 \\
\hline TOTAL & $\mathbf{1 , 0}$ & & $\mathbf{3 , 8}$ & & $\mathbf{3 , 9}$ & & $\mathbf{3 , 0}$ \\
\hline
\end{tabular}

Em cor vermelha é apresentado o amplificador escolhido para este processamento, sendo que o componente ótimo para a aplicação, neste caso foi de 3,9 obtido para o INA114. O termômetro foi escolhido para adquirir a temperatura corporal na axila. A seleção foi feita entre três termômetros: LM35, LM135 e AD592AN. Os critérios foram: sensibilidade, precisão, corrente de consumo e faixa da mensuração. Os pesos para cada critério foram definidos pela aplicação em biotelemetría e o local da medida. $\mathrm{Na}$ Tabela 2, são apresentados os critérios de seleção, os pesos e os resultados para cada componente. $\mathrm{O}$ maior valor somado definiu o componente ótimo para a aplicação, neste caso foi de 3,9 obtido para o LM35. 
Tabela 2: Parâmetros escolha sensor AI.

\begin{tabular}{|l|c|c|c|c|c|c|c|}
\hline \multicolumn{1}{|c|}{ SENSOR } & $\mathrm{Vr}$ & \multicolumn{2}{c|}{$\mathrm{LM} 35$} & \multicolumn{2}{c|}{ LM135 } & \multicolumn{2}{c|}{ AD592AN } \\
\hline \multicolumn{1}{|c|}{ Critério } & $\mathbf{\%}$ & Valor & Peso & Valor & Peso & Valor & Peso \\
\hline Sensibilidade & 30 & $10 \mathrm{mV} /{ }^{\circ} \mathrm{C}$ & 1,2 & $10 \mathrm{mV} /{ }^{\circ} \mathrm{K}$ & 0,3 & $1 \mathrm{mV} /{ }^{\circ} \mathrm{K}$ & 0,3 \\
\hline Precisão & 30 & $0.5^{\circ} \mathrm{C}$ & 1,2 & $1{ }^{\circ} \mathrm{C}$ & 0,9 & $0.5^{\circ} \mathrm{C}$ & 1,2 \\
\hline $\mathrm{I}_{\text {consumo }}$ & 10 & $10 \mathrm{~mA}$ & 0,3 & $15 \mathrm{~mA}$ & 0,2 & $298,2 \mu \mathrm{A}$ & 0,4 \\
\hline Faixa & 30 & $-55^{\circ} 150^{\circ} \mathrm{C}$ & 1,2 & $-55150^{\circ} \mathrm{C}$ & 1,2 & $-25105^{\circ} \mathrm{C}$ & 1,2 \\
\hline TOTAL & $\mathbf{1 , 0}$ & & $\mathbf{3 , 9}$ & & $\mathbf{2 , 6}$ & & $\mathbf{3 , 0}$ \\
\hline
\end{tabular}

Para o acelerômetro foram analisados três acelerômetros: MMA7455L, MMA7660FC e MMA7260Q. Os critérios foram: sensibilidade, tipo de saída, corrente de consumo e dimensão. Os pesos para cada critério foram definidos pela aplicação em biotelemetría e a localização no corpo. Na Tabela 3, são apresentados os critérios de seleção, sendo que para medir o deslocamento corporal foi escolhido o MMA7260Q, com 3,1.

Tabela 3: Parâmetros escolha acelerômetro.

\begin{tabular}{|l|c|c|c|c|c|c|c|}
\hline \multicolumn{1}{|c|}{ SENSOR } & Vr & \multicolumn{2}{c|}{ MMA7455L } & \multicolumn{2}{c|}{ MMA7660FC } & \multicolumn{2}{c|}{ MMA7260Q } \\
\hline \multicolumn{1}{|c|}{ Critério } & $\mathbf{\%}$ & Valor & Peso & Valor & Peso & Valor & Peso \\
\hline Sensibilidade & 30 & $2 / 4 / 8 \mathrm{~g}$ & 0,6 & $\pm 1,5 \mathrm{~g}$ & 0,3 & $1,5 / 2 / 4 \mathrm{~g}$ & 1,2 \\
\hline Saida & 20 & 8 bits & 0,6 & 6 bits & 0,4 & $800 \mathrm{mV}$ & 0,6 \\
\hline $\mathrm{I}_{\text {consumo }}[\mu \mathrm{A}]$ & 30 & 490 & 0,9 & 10 & 1,2 & 500 & 0,9 \\
\hline Dimensão $[\mathrm{mm}]$ & 20 & $3 \times 5 \times 1$ & 0,6 & $3 \times 3 \times 0,9$ & 0,8 & $6 \times 6 \times 1,45$ & 0,4 \\
\hline TOTAL & $\mathbf{1 , 0}$ & & $\mathbf{2 , 7}$ & & $\mathbf{2 , 7}$ & & $\mathbf{3 , 1}$ \\
\hline
\end{tabular}

\section{MÉTODO}

- Pré-Processamento dos Sinais - Nesta parte, precisam-se transformar os dados adquiridos. Para isto foi necessário desenvolver um circuito amplificador, logo filtrar e finalmente um circuito de acoplamento das impedâncias.

- Desenvolvimento da placa de pré-processamento e transmissão - São duas condições iniciais para o desenvolvimento da placa: a portabilidade (pequena, leve e confortável) e a livre mobilidade e autonomia (com a fonte de baterias externas e adaptáveis). A Figura 1 ilustra o esquema geral da placa.

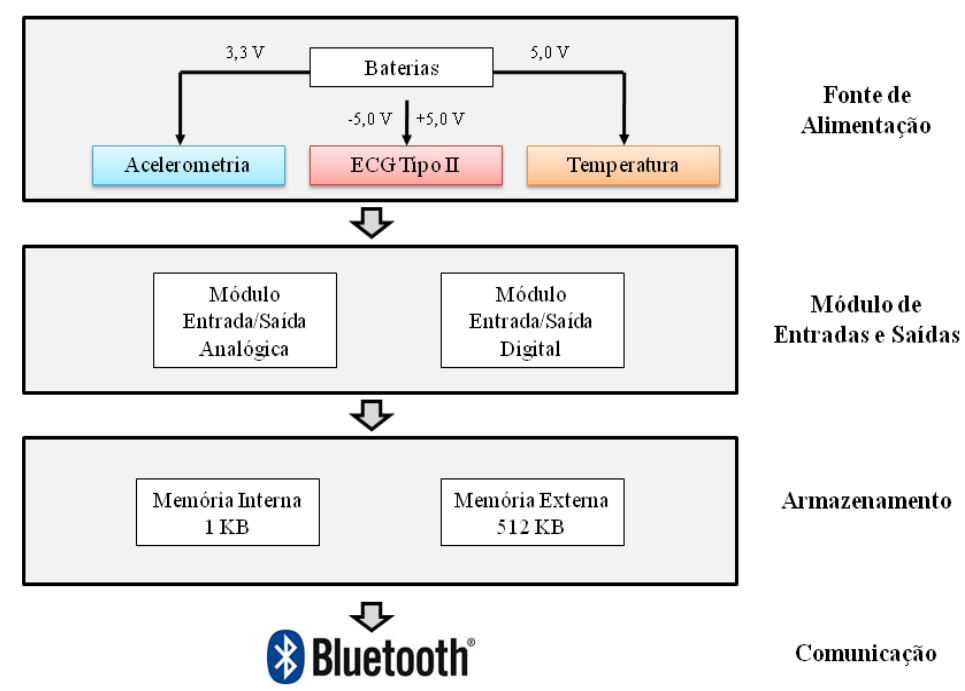

Figura 1 - Pré-processamento e transmissão 
- Fonte de alimentação Baterias - O Sistema de Biotelemetria proposto é energizado por duas baterias de Li-ion referência BB13-JV023-A, com capacidade de 7,2 V x 1000 $\mathrm{mAh}-7,2 \mathrm{Wh}$, peso de 53 gramas e 7,1x5,1x1,1 cm de dimensão. Para manter o circuito energizado, capturar os sinais, pré-processar os dados e armazenar na memória EEPROM externa o consumo de corrente medido no Sistema de Biotelemetria proposto é de $64 \mathrm{~mA}$. Quando se estabelece a comunicação do módulo com o PC via Bluetooth ${ }^{\mathrm{TM}}$ e inicia a transmissão dos dados a corrente de consumo medida se eleva para $120 \mathrm{~mA}$. Para garantir a funcionalidade do sistema foi desenvolvido um circuito para informar que a bateria descarregou, entrou em estado critico e que devem ser recarregadas.

- Módulo de entrada e saída dos dados - Tem a função de estabelecer comunicação entre o sistema de biotelemetria e o computador central pela porta Bluetooth ${ }^{\mathrm{TM}}$. O módulo está incorporado na placa Arduino® de forma a garantir a portabilidade e os protocolos da transmissão de dados. A Figura 2 ilustra a placa utilizada como módulo de entrada/saída de dados.

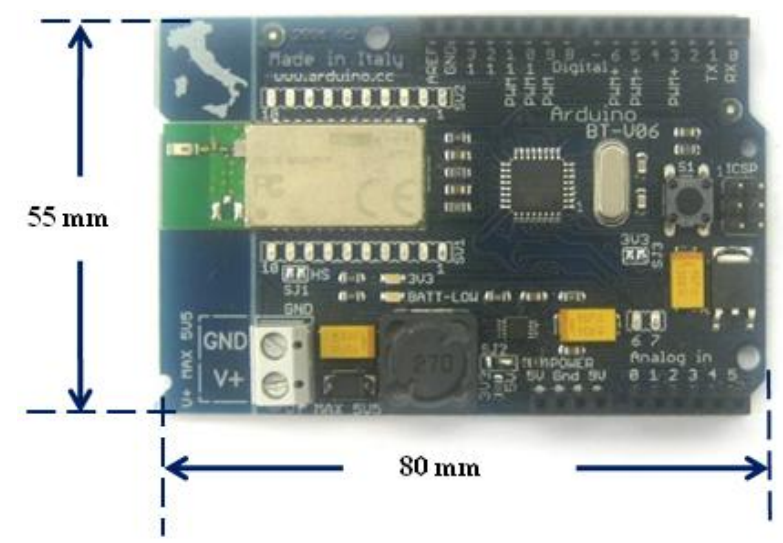

Figura 2 - Módulo de entrada e saída de dados

- Módulo de armazenamento dos dados - O módulo utiliza a memória EEPROM do Arduino ${ }^{\circledR}$ de 512 Bytes, quando à transmissão for online. Em virtude da necessidade de armazenamento dos dados durante atividades livres que sejam realizadas fora da faixa de captação de Bluetooth, foi desenvolvido um circuito externo com outra memória EEPROM de 1024 Bytes. As duas memórias, a externa e interna, foram usadas de acordo com dois critérios: tempo de monitoramento e área de cobertura da tecnologia Bluetooth.

- Programação dos módulos - A programação das aplicações foi em ambiente de desenvolvimento interativo para Arduino ${ }^{\circledR}$ (IDE), o que é uma aplicação multiplataforma escrita em Java. Inclui um editor de código com recursos de realce de sintaxe e biblioteca Wiring para programar em $\mathrm{C} / \mathrm{C}++$. A plataforma foi baseada em Atmel da AVR (ATMega168). Na Figura 3 podem-se ver as funcionalidades do programa usado. 


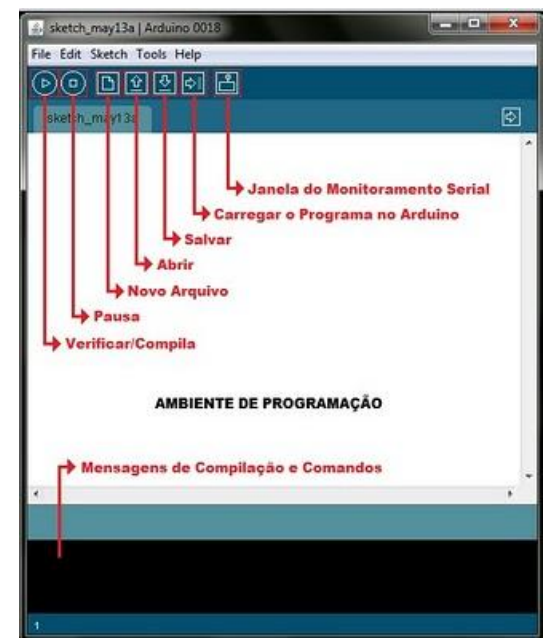

Figura 3 - Interface de programação

- Protocolo experimental de validação - A validação de aplicação de detecção de quedas foi feita com testes do acelerômetro triaxial, para detectar a queda de um indivíduo e analisar os valores limiares para detecção da queda em três diferentes situações.

- Amostra: um indivíduo saudável que simule três diferentes situações de queda.

- Instrumentação: Sistema de biotelemetria proposto com sensor acelerômetro triaxial.

Coleta de dados: Os dados foram coletados durante três estágios: i) queda do indivíduo considerando o mesmo em posição inicial de pé, ii) pulo de um patamar superior para um patamar inferior e iii) queda do indivíduo considerando o mesmo em posição inicial sentado. A coleta de dados foi continua antes e após a detecção da queda.

Teste experimental: Foi instalado no cinto do indivíduo voluntário o sistema de biotelemetria e se realizou três testes de queda: i) Indivíduo em pé com queda livre em direção ao chão, ii) Indivíduo pula de uma cadeira e de uma mesa em direção ao chão e iii) Indivíduo em posição inicial sentado com intenção de queda ao chão.

\section{RESULTADOS}

- Placa eletrônica de pré-processamento - Esta placa tem como função, fornecer energia para alimentação dos circuitos, adquirir os sinais fisiológicos, eliminar ruídos e entregar ao módulo de recepção e transmissão os dados que representem os sinais desejados. A Figura 4 ilustra a placa final

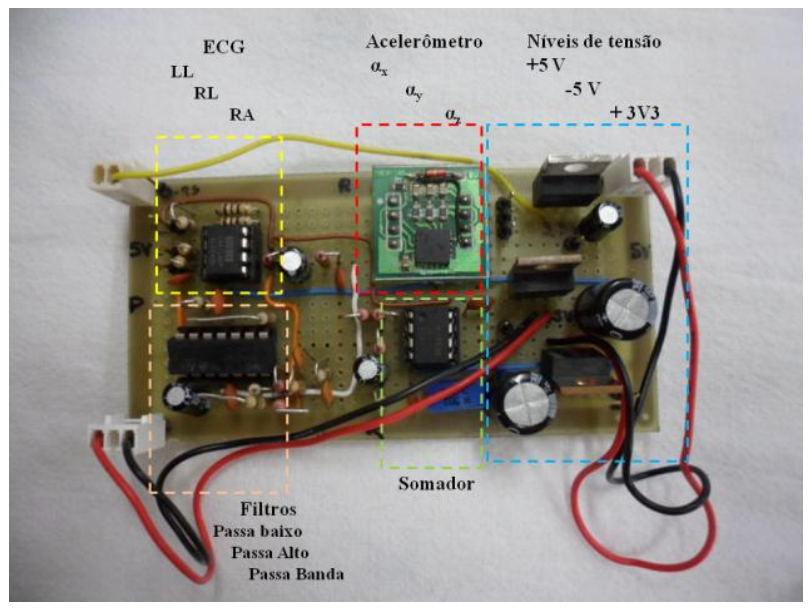




\section{Figura 4 - Placa final de Pré-Processamento}

- Aceleração corporal - As medidas da aceleração do corpo foram quantificadas em três eixos: $\alpha \mathrm{x}, \alpha y$ e $\alpha z$, avaliadas pela taxa de sensibilidade programada para os testes (faixa de $1,5 \mathrm{~g}$ sensibilidade $800 \mathrm{mV} / \mathrm{g}$ ). Os componentes cinemáticos foram: $\alpha \mathrm{x}$, negativa em sentido de esquerdo-direita; $\alpha y$, negativa em sentido de inferior-superior; e $\alpha z$, negativa em sentido de antero-posterior. Para o sensor acelerômetro a tensão de saída foi adaptada com relação ao zero-g offset, e a medida do ponto meio da tensão de alimentação 3,3 V. Portanto, os valores acima de 1,65 V foram considerados negativos e valores de 1,65 V foram considerados positivos, com relação à terra. Com exceção da tensão do eixo y que foi ajustada a $2,45 \mathrm{~V}$, resultado do cálculo da força de gravidade da terra de $1,5 \mathrm{~g}=800 \mathrm{mV} / \mathrm{g}$ em sentido vertical, assim: Vsaída $=1,65+0,8=2,45 \mathrm{~V}$. Os resultados do ajuste do acelerômetro são apresentados na Tabela 4.

Tabela 4: Ajuste do sensor acelerômetro

\begin{tabular}{|l|c|l|l|}
\hline DESLOCAMENTO & \multicolumn{3}{|c|}{ Acelerômetro } \\
\hline \multicolumn{1}{|c|}{ Posição } & $\mathrm{x}[\mathrm{g}]$ & \multicolumn{1}{c|}{$\mathrm{y}[\mathrm{g}]$} & $\mathrm{z}[\mathrm{g}]$ \\
\hline Inicial & $0.33 \pm 0.002$ & $0.94 \pm 0.003$ & $0.056 \pm 0.003$ \\
\hline à direita & $1.01 \pm 0.009$ & $0.034 \pm 0.008$ & $0.34 \pm 0.008$ \\
\hline à esquerda & $-0.98 \pm 0.007$ & $0.062 \pm 0.007$ & $0.053 \pm 0.007$ \\
\hline para abaixo & $0.015 \pm 0.005$ & $1.64 \pm 0.05$ & $0.047 \pm 0.005$ \\
\hline
\end{tabular}

Desenho final - O desenho do sistema de biotelemetria é apresentado na Figura 5. A caixa tem duas entradas para o carregador de baterias externo, dispositivo de $16,8 \mathrm{~V}$ configuradas em série. O LED vermelho é o sinalizador de bateria fraca que piscará no instante em que as baterias atingirem $80 \%$ de utilização Pré-Processamento.

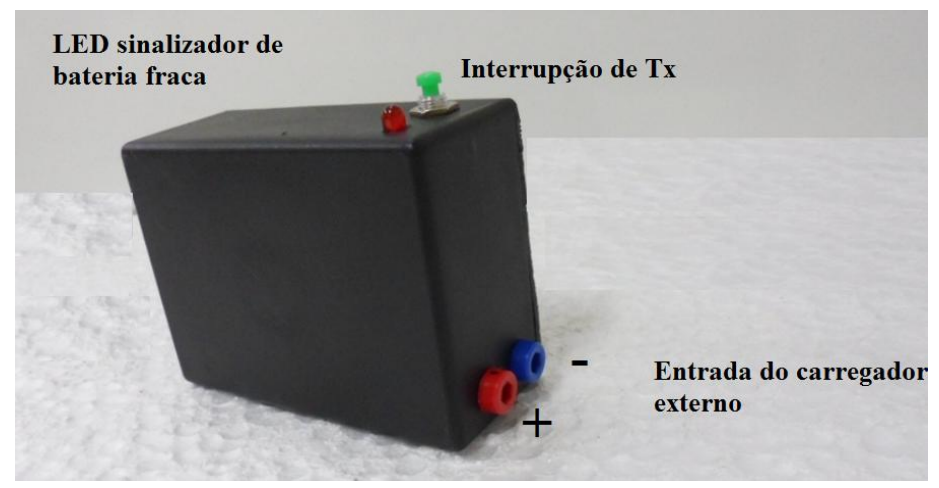

Figura 5 - Desenho final

\section{Aplicação: detecção de quedas}

Uma queda pode causar desde lesões físicas e mentais até a morte, pelo tardio atendimento ao paciente. No Brasil, indivíduos com 60 anos ou mais representavam 5,0\% da população geral, na década de 40 e segundo o Instituto Brasileiro de Geografia Estatística (IBGE) a população de idosos passou de 6,1\% em 1980 para 7,3\% em 1991. Estima-se que em 2025 essa população seja de 14,0\% [5]. 
A queda pode ser definida como evento em que a pessoa inadvertidamente vai ao solo ou outro local em nível mais baixo do que o anteriormente ocupado, com ou sem perca de consciência ou lesão [6,7], ou como a falta de capacidade para corrigir o deslocamento do corpo durante o movimento no espaço $[8,9]$.

Tendo como referência que a queda é decorrente da perca total do equilíbrio postural, a mensuração desse deslocamento por meio do sistema de biotelemetria, usando o acelerômetro triaxial, permite detectar a queda em tempo médio de $50 \mathrm{~ms}$. A Figura 6 apresenta a sinal da aceleração triaxial durante o teste do indivíduo em pé com queda livre em direção ao chão.

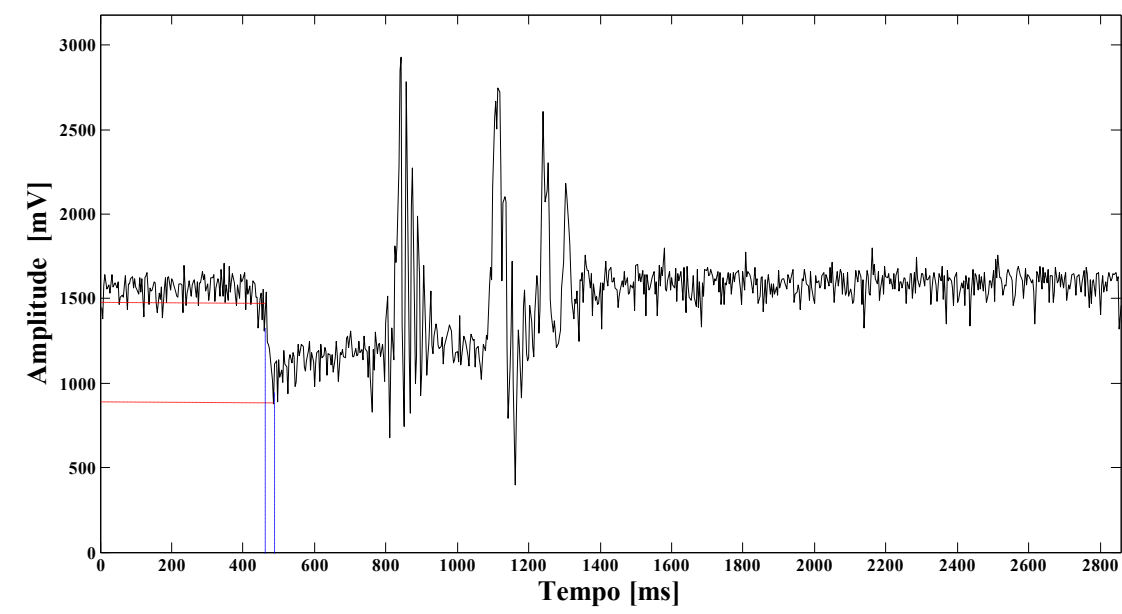

Figura 6 - Sinal de aceleração triaxial durante queda controlada

Nota-se que a queda foi detectada pela diferença brusca no nível de voltagem $1500 \mathrm{mV}$ para $960 \mathrm{mV}$. O sensor acelerômetro utilizado foi programado com sensibilidade de 1,5 g $(800 \mathrm{mV})$. Na Figura 7 foi amplificado o evento da queda.

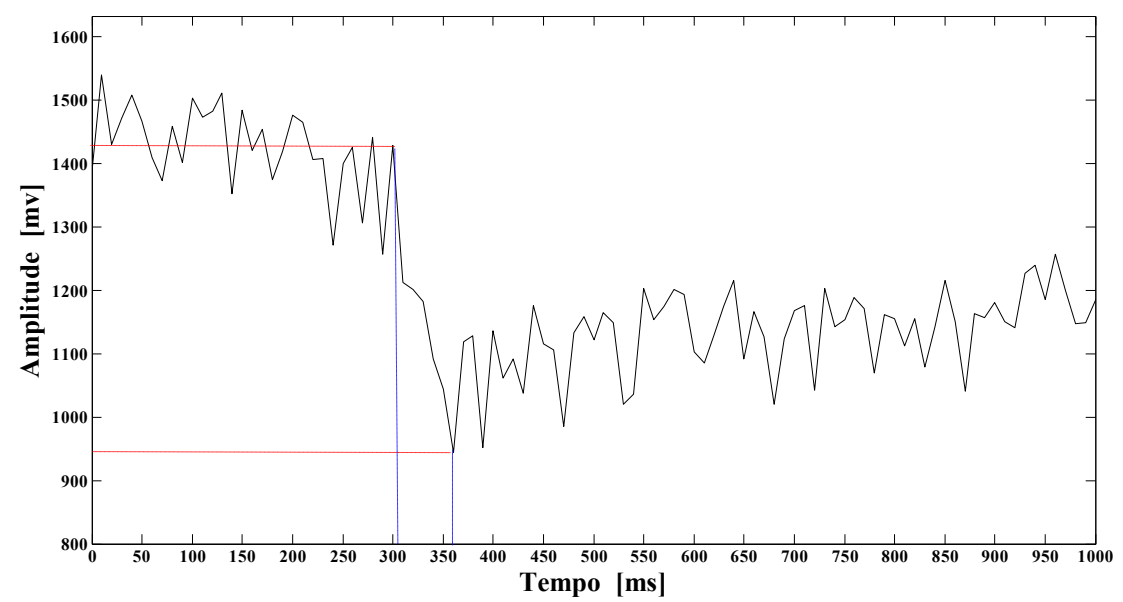

Figura 7 - Detalhe da detecção da queda

Para detectar uma queda usando o sistema de biotelemetria devem-se cumprir obrigatoriamente duas condições: i) Uma queda de $-530 \mathrm{mV}$ do vetor magnitude da aceleração, correspondente aprox. a $1 \mathrm{~g}$. ii) A queda anterior deve acontecer em tempo menor a $50 \mathrm{~ms}$. Para confirmar estas duas condições foram feitos dois testes adicionais, um fazendo o indivíduo pular de uma cadeira e de uma mesa sendo que o sistema de detecção conseguiu 
cumprir as duas condições. Outro teste foi desde uma posição sentada, o indivíduo faz intenção de queda, neste caso a queda foi menor a $530 \mathrm{mV}$ e a primeira condição não foi realizada.

\section{DISCUSSÃO}

Este novo sistema de biotelemetría permite trazer muito mais benefícios de automonitoramento das variáveis que aumentam os fatores de risco para doenças não transmissíveis. Para tanto nesta pesquisa foi necessário e tornou-se imperativo que as novas tecnologias usadas pudessem ser utilizadas com eficiência e segurança para populações afastadas dos avanços tecnológicos.

Com relação ao consumo de energia das baterias, em [9], estuda o consumo critico de sistemas não invasivos em monitoramento médico, ressaltando que o custo e a autonomia da bateria são fatores limitantes para o desenvolvimento desta tecnologia.

Neste trabalho o protótipo utiliza duas baterias recarregáveis de $9 \mathrm{~V} 320 \mathrm{mAh}$ para um consumo médio do sistema multicanal de $84 \mathrm{mAh}$ obtendo uma autonomia medida não maior a 3 horas com transmissão ativa. Concordando com [9] se propõe trocar as baterias atuais por baterias de 7,2 V/Li-ion $1000 \mathrm{mAh}$ as quais proporcionam uma melhoria de $300 \%$.

Para cumprir com os parâmetros de ergonomia que permitam o livre movimento e conforto do individuo, concordando com [10]. O protótipo apresentado $(15 \mathrm{~cm} \mathrm{x} 10 \mathrm{~cm})$ poderá ser redimensionado para uma placa de 7,5 cm x $5,5 \mathrm{~cm}$ similar a um telefone celular.

\section{CONCLUSÃO}

A validação dos sinais apresentou correlação considerada alta $r=0.8$ com $p<0,05$ entre o padrão e o alvo para o sinal mais complicado de processar. Estes resultados dos testes experimentais permitem apresentar um sistema de biotelemetría útil em áreas locais. O desenho da caixa é ergonômico, portável, leve e permite o livre deslocamento.

No Brasil, a população idosa, com aproximadamente 15 milhões de indivíduos, já representa mais de $9 \%$ da população. Esta população cresce muito mais rapidamente que a qualidade e a disponibilidade dos nossos ultrapassados serviços médico-assistenciais.

Diante de notável transformação epidemiológica que o Brasil vem sofrendo nestes últimos tempos, a qualidade de vida ganha um sentido mais amplo e necessário, criando-se a necessidade de um olhar mais crítico e intervenções a respeito das alterações do envelhecimento e de suas consequências, a fim de proporcionar ao idoso um maior bem-estar e maior capacidade funcional.

\section{REFERÊNCIAS BIBLIOGRÁFICAS}

1. AMERICAN GERIATRICS SOCIETY. Guideline for the prevention of falls in older persons. British Geriatrics Society, Academy of Orthopedic Surgeons Panel on falls Prevention. J Am Geriatr Soc. 2001; 49(5):664-72.

2. BASSET DR, AINSWORTH BE, SWARTZ AM, et al. Validity of four motion sensors in measuring moderate intensity physical activity. Med Sci Sports Exerc, 32: S471-80, 2000. 
3. BRITO, F.C, COSTA, S.M.N. Quedas. In: Papaléu Netto, M., Brito, F.C. Urgências em geriatria: epidemiologia, fisiopatologia, quadro clínico. Controle terapêutico. São Paulo, Rio de Janeiro, Belo Horizonte. Editora Atheneu, cap.26, p. 323, 2001.

4. CHUNG WY, LEE YD, JUNG SJ. A wireless sensor network compatible wearable u-healthcare monitoring system using integrated ECG, accelerometer and SpO2. In Proc IEEE Eng Med Biol Soc; 2008:1529-32.

5. COOK DJ, AUGUSTO JC E JAKKULA VR. Ambient intelligence: technologies, applications, and opportunities, Pervasive and Mobile Computing 5 (August) (2009) 277-298.

6. ICHIKAWA, FC; CHIPCHASE, J; GRIGNANI, R. Where's The Phone? A Study of Mobile Phone Location in Public Spaces. In: Proceedings International Conference Mobile Technology, Applications and Systems, 2., p.1-8:15-17, 2005.

7. JUNG J., HA K., AND LEE J., KIM Y. AND KIM D. Wireless body área network in a ubiquitous healthcare system for physiological signal monitoring and health consulting. Int J Signal Proc and Pat Recog, 2008 (11), 47-54.

8. LUPU RG, STAN A E UNGUREANU F. Wireless Device for Patient Monitoring. In Proc World Congress on Engineering. 2008 (2), London, U.K.

9. MATHIE, MJ, CELLER BG, COSTER ACF, LOVELL NH. Classification of basic daily movements using a triaxial accelerometer. Medical \& Biological Engineering \& Computing, Vol.42, 2004, pp.679-687.

10. MISHRA S, NAYAK CG, SHET KC, GEORGE VI. Telecommunication technology used in home healthcare. J Indian Med Assoc. 2011 Jan;109(1):38-9.

11. MUNDT CW, MONTGOMERY KN, et al. A multiparameter wearable physiologic monitoring system for space and terrestrial applications. IEEE Trans Inf Technol Biomed. 2005 Sep; 9(3):382-91.

12. ORGANIZAÇÃO MUNDIAL DA SAÚDE. Document, WHO/DGO/98.01, A Health Telematics policy: in support of the WHO's Health-for-All strategy for global health development, report of the WHO Group Consultation on Health Telematics, 11-16, December, Geneva, 1997.

13. VAN HEES VT, VAN LUMMEL RC, E WESTERTERP K. Estimating Activityrelated Energy Expenditure Under Sedentary Conditions Using a Tri-axial Seismic Accelerometer. Obesity (Silver Spring), 17(6):1287-92, 2009.

14. VELÁZQUEZ ALVA, M. C. Nutrition in Geriatrics. Advanced Course in Clinical Nutrition. The International Confederation for Nutrition Support Organizations. 2008. Disponível

em: $<$ http://http://www.nutritotal.com.br/icnso/aulas/?acao=bu\&categoria=57\%>. Acesso em: 25 set. 2010.

15. WAC K, BULTS R, VAN BEIJNUM B, et al. Mobile patient monitoring: the MobiHealth system. Conf Proc IEEE Eng Med Biol Soc. 2009;2009:1238-41. 